\title{
Postgraduate trainees' perceptions of the learning environment in a Nigerian teaching hospital
}

\author{
P I Idon, ${ }^{1}$ BDS, FMCDS; I K Suleiman, ${ }^{1}$ BDS; H O Olasoji, ${ }^{1,2}$ BSc, BChD, FMCDS, FWACS, MSc Med Educ (UK); Z Mustapha, ${ }^{3}$ MBBS, FWACS; \\ H M Abba, ${ }^{1}$ BDS
}

${ }^{1}$ Department of Dental Surgery, University of Maiduguri Teaching Hospital, Nigeria

${ }^{2}$ Department of Oral and Maxillofacial Surgery and Oral Pathology, Faculty of Dentistry, College of Medical Sciences, University of Maiduguri, Nigeria

${ }^{3}$ Department of Radiology, College of Medical Sciences, University of Maiduguri Teaching Hospital, Nigeria

Corresponding author: P I Idon (idonp85@gmail.com)

Background. The learning environment represents various factors that describe the learner's experiences in that setting. The learning environment of junior doctors undergoing training programmes in hospitals is considered a major factor determining both academic success and health service delivery performance. Increased performance in both areas requires routine assessment of the learning environment to identify components that need attention.

Objective. To evaluate the perception of junior doctors undergoing specialist training regarding the learning environment in a teaching hospital. Methods. This was a single-centre, cross-sectional study, using the Postgraduate Hospital Educational Environment Measure (PHEEM). The questionnaire was used to collect data on the learning environment of junior doctors in all 10 clinical departments at the University of Maiduguri Teaching Hospital, Nigeria. All of the junior doctors $(n=148)$ in the hospital at the time of the study received the questionnaire; they constituted the sample size for the survey. Data collected were analysed to assess junior doctors' perceptions of the overall learning environment and of the individual factors in the learning environment as measured by the individual items of PHEEM.

Results. The hospital educational environment was rated high, with a score of 98.25. The domains of the environment measure also showed positive perceptions, but revealed specific areas in need of attention as measured by the items of the questionnaire. Significant $(p<0.05)$ differences were noted in the perceptions of some items of the environment in the clinical departments.

Conclusions. The junior doctors' perceptions of their educational environment were positive. The study was able to identify areas of strengths and weaknesses in the overall hospital learning environment and the specialty departments. Overall, it identified the absence of an informative handbook for junior doctors and quality accommodation and catering facilities when the doctors were on call, as well as excess workload and lack of counselling services as areas that require the most attention to improve the learning environment.

Afr J Health Professions Educ 2017;9(3):116-122. DOI:10.7196/AJHPE.2017.v9i3.786

The West African College of Surgeons (WACS), West African College of Physicians (WACP) and the National Postgraduate Medical College of Nigeria (NPMCN) regulate the postgraduate medical and dental education in the West African region and Nigeria, respectively. ${ }^{[1,2]}$

Currently, progress report forms are completed annually by the trainers, i.e. specialist physicians and surgeons in the various teaching hospitals, to assess the progress of postgraduate training of junior doctors. At regular intervals, the colleges visit these hospitals to assess the facilities for postgraduate training for the purpose of maintaining the standard of training and re-accreditation of the institution for continued training of junior doctors. The term junior doctor in this regard refers to all cadres of doctors, except specialist physicians or surgeons who are fellows of any of the postgraduate medical colleges. These include senior resident doctors, junior resident doctors, medical officers, senior house officers and house officers. The resident doctors are those undergoing the residency training programme in various specialties to become specialist physicians or surgeons under the supervision of consultants. Medical officers, although not enrolled in the residency training programme, also work under and learn from the specialists. The senior house officers and house officers are junior doctors at different stages of their pre-registration and compulsory supervised training after graduation from medical school. The specialist physicians and surgeons are appointed as consultants in the teaching hospitals to consult and oversee the training of junior doctors. To date, these assessments only focused on the trainees' progress and did not take into consideration their perceptions of the trainers and other components in the environment in which they work, and how these affect their learning. The learning environment or educational environment has been considered to encompass physical (safety, food, shelter, comfort), emotional (security, feedback from trainers, absence of bullying and harassment) and intellectual (learning with patients, relevance to practice, evidence-based, active participation by learners) aspects. ${ }^{[3]}$

Working and learning in a clinical environment represent a challenging phase for doctors in training. Junior doctors in this environment have to achieve a balance between a myriad of things, including care for their patients, adhering to their work schedules, dealing with the loss of a patient, and continuing with their academic pursuits, along with an obligation to their family, and their personal life. ${ }^{[4]}$ The type or quality of the learning environment in a teaching hospital affects the motivation for clinical 
training, knowledge base and performance of junior doctors. ${ }^{[5,6]}$ The teaching hospital has the responsibility of providing satisfactory education for its junior doctors, with the aim of improving the services they render to the public. ${ }^{[7]}$ Since recognising the importance of the quality of the learning environment of teaching hospitals in postgraduate medical education, the topic has received increased attention in the literature. ${ }^{[8-10]}$ Problems of junior doctors in the learning environment have been noted to include lack of clear objectives regarding the curriculum and its scope. The focus has been on knowledge acquisition, with little emphasis on problem-solving skills. Other problems noted have included high workload, leaving little time for academic activities, as well as the more talked-about teaching-byhumiliation method employed by trainers. ${ }^{[1]]}$

Roff $^{[12]}$ constructed and validated the Postgraduate Hospital Educational Environment Measure (PHEEM) for hospital-based junior doctors in the UK. This tool is considered reliable for evaluating the quality of the educational environment of teaching hospitals, and has been used to measure the educational environment for junior doctors in several countries. $^{[5,6,12,13]}$ The lack of empirical data means that little is known about the way junior doctors perceive their learning environment. Also, it provides an additional needed input on facilities and learning environment as perceived by the trainees. Postgraduate regulatory bodies may use this as part of the assessment tools for the accreditation and re-accreditation of teaching hospitals for the training of junior doctors. The aim of this study was therefore to evaluate the perceptions of junior doctors regarding the learning environment at the University of Maiduguri Teaching Hospital (UMTH), Nigeria, by using PHEEM. The results of this study can serve as a basis for improvement and for future evaluation/comparison of trainees' perceptions of Nigerian postgraduate medical and dental education.

The study therefore set out to determine:

- the junior doctors' perceptions of the hospital learning environment at UMTH

- the effect of area of specialty on the perceptions of the learning environment

- the effect of gender on the perceptions of the learning environment.

\section{Methods \\ Study setting}

The study was conducted at UMTH, which is the main tertiary health centre in North-Eastern Nigeria. It is located in Maiduguri, a semi-urban settlement and the capital of Borno State. The hospital serves as a major referral centre for the North-Eastern states and as a training centre for junior doctors in several specialties. Currently, the hospital carries out training of junior doctors in 10 specialty departments: dental surgery, general outpatients, ophthalmology, ear nose and throat, internal medicine, obstetrics and gynaecology, paediatrics, pathology, radiology and surgery.

\section{Study design and recruitment of participants}

The study employed a cross-sectional research approach in assessing the perceptions of the participants with regard to the hospital learning environment. Employing a census survey method of sampling, all doctors below the grade rank of consultant in the various hospital departments and specialties at the time of the study in 2014 were included and constituted the study population. These included the resident doctors, medical officers, senior house officers and house officers. The participants were recruited individually into the study in their departments following an introduction of the objective of the study.

\section{Data collection method}

The study used two self-administered questionnaires to collect data from the participants. The first consisted of short structured questions constructed to collect demographical information, such as the participant's gender, age group, training grade, year in current grade and specialty. Information on the participant's perception of the hospital's learning environment was collected with the PHEEM questionnaire. The researchers distributed questionnaires to all the participants by hand and retrieved the completed questionnaires in the same way. To achieve confidentiality, the data obtained from the participants did not include their names and therefore cannot be linked to any individual participant.

PHEEM, as an assessment tool for the learning environment, is simple and practical, taking $<5$ minutes to complete, and has been validated in several studies, with reliability values of 0.92 and 0.93 using Cronbach's alpha. ${ }^{[6,12,13]}$ The questionnaire consists of 40 items with regard to learning environment, divided into three subscales, i.e. perception of role autonomy; perception of teaching; and perception of social support. Responses to each statement were indicated on a 5-point Likert scale as follows: 0 for strongly disagree, 1 for disagree, 2 for uncertain, 3 for agree and 4 for strongly agree. The maximum possible score is 4 or 160 and the minimum is 0 for item score and overall scores, respectively, with higher scores indicating a better educational environment. Four of the 40 items (items 7, 8, 11 and 13) are negative statements and were scored in reverse. Three of the items were modified to suit the context in which the study was being carried out. Item 7 ('There is racism in this post') was not applicable, as most of the junior doctors are Nigerians, but of different tribes. The item was therefore modified to read, 'There is racism/tribalism in this post'. Item 11 ('I am bleeped inappropriately') was also modified because junior doctors are either required to stay in the emergency department or call rooms when on duty, or are fetched at their residence in the hospital quarters when the need arises. This was modified to read, 'I am called inappropriately'. Also, item 17 was modified to read, 'My hours of work conform to the civil service rule', as applicable in the country.

\section{Data analysis}

Analysis of the data obtained was performed using the Statistical Package for Social Sciences (SPSS) version 17.0 (SPSS Inc., USA). Descriptive statistics were reported in the form of frequencies, percentages, means, and standard deviations (SDs). Student's $t$-test was used to compare the item mean and overall PHEEM scores between the genders, while analysis of variance (ANOVA) statistics was used to analyse mean scores of items and overall scores among the training grades and specialties. Statistical significance was inferred at $p<0.05$.

\section{Ethical approval}

The Research and Ethical Committee of the hospital approved the study (ref. no. UMTH/REC/17/0089) before commencement. A detailed explanation was given to each participant, with assurance of confidentiality regarding data collection and analysis. To achieve such confidentiality, names of participants were not included in the data collected. In data analysis, participants were assured that information provided will be deidentified by aggregating responses of individuals into groups and reporting them as means and SDs. Participation was voluntary, with consent sought and obtained from each participant included in the study.

\section{Results}

Of the 148 participants, 108 (66 males and 42 females) completed and returned the questionnaires - a response rate of $73 \%$. Data were received 
from all 10 specialty departments of the hospital. The highest response rates were recorded from the ophthalmology, radiology and dental specialties, and the lowest rate $(50 \%)$ from the surgery specialty. The number of junior doctors in the specialty areas ranged from 2 to 17 , distributed among the various cadres of junior doctors, house officers, senior house officers, medical officers, junior registrars and senior registrars (Table 1).

The mean scores and SDs of the 40 items of the PHEEM questionnaire are shown in Table 2. The lowest item score was 1.08 for item 26 ('There are adequate catering facilities when I'm on call'), while the highest was 3.16 ('I have good collaboration with other doctors in my grade'). Items 9 and 32 were scored less than average (2.0) in the perception of the autonomy domain. Items 20,26 and 38 , relating to social support, were also $<2.0$. These items indicate problem areas in the learning environment. All other items had scores within the range of 2 - 3 ('A more supportive/suitable educational environment, but with need for enhancement'), while only item 29 ('I feel part of a team working here') in the autonomy section and items 7, 13 and 16 in the social support section had scores $>3.0$.

An overall score of 98.25 was obtained, with a score of 34.52 for perception of autonomy, 37.91 for perception of teaching and 25.76 for perception of social support (Table 3). Also shown in Table 3 are the various domain scores and overall scores for the different specialties. More items were reported with below-average scores in the obstetrics and gynaecology and paediatrics specialties, while dental surgery and ophthalmology did not score less than average for any of the items (Table 4).

Table 1. Demographic distribution of the participants $(N=108)$

\begin{tabular}{ll}
\hline Demographic distribution & Frequency (\%) \\
\hline Gender & $66(61.1)$ \\
Male & $42(38.9)$ \\
Female & $108(100)$ \\
Total & \\
Training level & $26(24.1)$ \\
House officer & $5(4.6)$ \\
Senior house officer & $22(20.4)$ \\
Medical officer & $36(33.3)$ \\
Junior registrar & $19(17.6)$ \\
Senior registrar & $108(100)$ \\
Total & \\
Specialty & $16(14.8)$ \\
Dental surgery & $14(13.0)$ \\
Medicine & $10(9.3)$ \\
Obstetrics and gynaecology & $9(8.3)$ \\
Pathology & $17(15.7)$ \\
Paediatrics & $10(9.3)$ \\
Surgery & $14(13.0)$ \\
Radiology & $2(1.9)$ \\
Ophthalmology & $3(2.8)$ \\
Ear, nose and throat & $13(12.0)$ \\
General outpatient department & $108(100)$ \\
Total &
\end{tabular}

Using Kruskal-Wallis (data normally distributed) one-way analysis of variance (ANOVA), comparison of these scores among the specialties and the training grades did not show any statistically significant difference, with $p=0.055$ and $p=0.478$, respectively. The Mann-Whitney $U$-test for domain (perception of role autonomy, $p=0.796$; perception of teaching, $p=0.186$; perception of social support, $p=0.867$ ) and overall scores between the genders also did not show statistically significant differences $(p=0.592)$. However, differences were noticed using one-way ANOVA in item scores among the specialties (Table 5). Post-hoc analysis using the Tukey honest significance difference (HSD) (equal variance assumed) and Games-Howell (equal variance assumption not met) statistics revealed the specialties with significant differences.

Junior doctors in radiology had a significantly better perception (mean 3.29 (SD 0.47)) of the appropriate level of responsibility (item 5) compared with those in internal medicine (2.14 (1.01)). Dental surgery (1.19 (0.98)) and internal medicine (1.21 (1.12)) specialties rated item 9 significantly lower than obstetrics and gynaecology $(2.80(0.92))$. The doctors in radiology $(3.07$ $(0.73))$ and obstetrics and gynaecology $(3.50(0.71))$ gave significantly better ratings to item 14 than respondents from internal medicine (1.86 (1.09)), dental surgery $(2.19(1.11))$ and paediatrics $(2.12(0.93)$.

The perceptions of junior doctors in ophthalmology $(3.00(0.00))$ and radiology $(3.14(0.77))$ about their hours of work conforming to the civil service rule were significantly better than those of their colleagues in internal medicine $(2.00(0.96))$ and surgery $(1.10(0.88))$. Item 26 revealed a statistically significant difference among the specialties $(p=0.013)$. A posthoc Tukey HSD test revealed a significant difference between respondents in radiology $(2.00(1.24))$ and those in obstetrics and gynaecology $(0.50$ $(0.71))$ and surgery $(0.60(0.52))$ regarding the quality of the catering service while on call.

Paediatric (1.29 (1.21)) and obstetrics and gynaecology (0.60 (1.00)) specialties had a greater level of disagreement with item 32 ('My workload in this job is fine') compared with the response from dental surgery $(2.81$ (1.05)), pathology $(2.78(0.67))$, radiology $(2.79(0.80))$ and ophthalmology (3.00 (0.00)). Respondents from ophthalmology $(3.00(0.00))$ rated the mentoring skills of their teachers significantly higher than the junior doctors in paediatrics $(2.00(1.06))$ and there was also better feedback from them than from junior doctors in the general outpatient department (GOPD) (1.85 (0.99)).

\section{Discussion}

This study used PHEEM to assess the educational environment of junior doctors in a teaching hospital setting involving all the specialty departments. It also included junior doctors who are not yet in the residency programme, e.g. medical officers, senior house officers and house officers. From the results it was evident that PHEEM is a reliable tool for assessing the strengths and weaknesses of the postgraduate hospital training environment. ${ }^{[14]}$

The learning environment in the hospital was valued fairly well by the junior doctors, but with room for improvement, as shown by a score of 98.25, corresponding to the 'more positive than negative environment' according to the criteria proposed by PHEEM. ${ }^{[12]}$ The three subscale scores also revealed that there was a more positive perception towards the role of autonomy, and that the perceptions of teaching were moving in the right direction. Furthermore, it was found that the perceptions of social support had more positives than negatives.

The lowest recorded item score was 1.08 (item 26: 'There are adequate catering facilities when I am on call') and the highest was 3.16 (item 16: 'I 


\section{Research}

Table 2. Mean scores of each item of the PHEEM questionnaire

\begin{tabular}{|c|c|c|}
\hline Item & Domain & Mean (SD) \\
\hline \multicolumn{3}{|c|}{ Perception of role of autonomy } \\
\hline 1 & I have a contract of employment that provides information about hours of work & $2.20(1.17)$ \\
\hline 4 & I had an informative induction programme & $2.07(1.13)$ \\
\hline 5 & I have the appropriate level of responsibility in this post & $2.81(0.98)$ \\
\hline 8 & I have to perform inappropriate tasks & $2.38(1.15)$ \\
\hline 9 & There is an informative junior doctors' handbook & $1.75(1.08)$ \\
\hline 11 & I am called inappropriately & $2.56(1.05)$ \\
\hline 14 & There are clear clinical protocols in this post & $2.44(1.00)$ \\
\hline 17 & My hours of work conform to the civil service rule & $2.19(1.22)$ \\
\hline 18 & I have the opportunity to provide continuity of care & $2.86(0.63)$ \\
\hline 29 & I feel part of a team working here & $3.01(0.83)$ \\
\hline 30 & I have opportunities to acquire appropriate practical procedures for my grade & $2.81(0.83)$ \\
\hline 32 & My workload in this job is fine & $1.96(1.30)$ \\
\hline 34 & The training in this post makes me feel ready to be a senior registrar/consultant & $2.62(0.92)$ \\
\hline 40 & My clinical teachers promote an atmosphere of mutual respect & $2.82(0.98)$ \\
\hline \multicolumn{3}{|c|}{ Perception of teaching } \\
\hline 2 & My clinical teachers set clear expectations & $2.73(1.01)$ \\
\hline 3 & I have protected educational time in this post & $2.21(1.11)$ \\
\hline 6 & I have good clinical supervision at all times & $2.28(1.11)$ \\
\hline 10 & My clinical teachers have good communication skills & $2.94(0.86)$ \\
\hline 12 & I am able to participate actively in educational events & $2.82(0.91)$ \\
\hline 15 & My clinical teachers are enthusiastic & $2.80(0.83)$ \\
\hline 21 & There is access to an educational programme relevant to my needs & $2.16(1.06)$ \\
\hline 22 & I get regular feedback from seniors & $2.48(0.89)$ \\
\hline 23 & My clinical teachers are well organised & $2.51(1.05)$ \\
\hline 27 & I have enough clinical learning opportunities for my needs & $2.03(1.13)$ \\
\hline 28 & My clinical teachers have good teaching skills & $2.91(0.76)$ \\
\hline 31 & My clinical teachers are accessible & $2.77(0.97)$ \\
\hline 33 & Senior staff utilise learning opportunities effectively & $2.25(0.93)$ \\
\hline 37 & My clinical teachers encourage me to be an independent learner & $2.74(0.97)$ \\
\hline 39 & My clinical teachers provide me with good feedback on my strengths and weaknesses & $2.28(1.05)$ \\
\hline \multicolumn{3}{|c|}{ Perception of social support } \\
\hline 7 & There is racism/ tribalism in this post & $3.03(1.02)$ \\
\hline 13 & There is sex discrimination in this post & $3.07(1.04)$ \\
\hline 16 & I have good collaboration with other doctors in my grade & $3.16(0.63)$ \\
\hline 19 & I have suitable access to careers advice & $2.25(1.09)$ \\
\hline 20 & This hospital has good-quality accommodation for junior doctors, especially when on call & $1.79(1.24)$ \\
\hline 24 & I feel physically safe within the hospital environment & $2.69(0.98)$ \\
\hline 25 & There is a no-blame culture in this post & $2.06(1.09)$ \\
\hline 26 & There are adequate catering facilities when I am on call & $1.08(1.09)$ \\
\hline 35 & My clinical teachers have good mentoring skills & $2.53(0.97)$ \\
\hline 36 & I get a lot of enjoyment out of my present job & $2.29(1.00)$ \\
\hline 38 & There are good counselling opportunities for junior doctors who fail to complete their training satisfactorily & $1.90(1.18)$ \\
\hline
\end{tabular}




\section{Research}

Table 3. Subscale and overall PHEEM scores of the different specialties

\begin{tabular}{|c|c|c|c|c|c|c|c|c|c|c|c|c|}
\hline Score & Assessment & $\begin{array}{l}\text { Dental } \\
\text { surgery }\end{array}$ & $\begin{array}{l}\text { Medi- } \\
\text { cine }\end{array}$ & $O \& G$ & $\begin{array}{l}\text { Patho- } \\
\text { logy }\end{array}$ & $\begin{array}{l}\text { Paediat- } \\
\text { rics }\end{array}$ & Surgery & $\begin{array}{l}\text { Radio- } \\
\text { logy }\end{array}$ & $\begin{array}{l}\text { Ophthal- } \\
\text { mology }\end{array}$ & ENT & GOPD & $\begin{array}{l}\text { Mean } \\
(\mathrm{SD})\end{array}$ \\
\hline \multicolumn{13}{|c|}{ Perception of role of autonomy } \\
\hline $0-14$ & Very poor & & & & & & & & & & & \\
\hline $15-28$ & $\begin{array}{l}\text { A negative view of } \\
\text { one's role }\end{array}$ & & & & & & & & & & & \\
\hline $29-42$ & $\begin{array}{l}\text { A more positive } \\
\text { perception of one's } \\
\text { job }\end{array}$ & 35.1 & 30.4 & 36.0 & 38.1 & 32.2 & 32.2 & 40.6 & 36.5 & 32.7 & 32.9 & $\begin{array}{l}34.5 \\
(7.3)\end{array}$ \\
\hline $43-56$ & $\begin{array}{l}\text { Excellent } \\
\text { perception of one's } \\
\text { job }\end{array}$ & & & & & & & & & & & \\
\hline \multicolumn{13}{|c|}{ Perception of teaching } \\
\hline $0-15$ & Very poor & & & & & & & & & & & \\
\hline $16-30$ & $\begin{array}{l}\text { In need of some } \\
\text { training }\end{array}$ & & & & & & & & & & & \\
\hline $31-45$ & $\begin{array}{l}\text { Moving in the } \\
\text { right direction }\end{array}$ & 38.3 & 34.0 & 39.4 & 40.9 & 33.8 & 40.4 & 44.2 & 41.0 & 40.3 & 33.9 & $\begin{array}{l}37.9 \\
(8.8)\end{array}$ \\
\hline $46-60$ & Model teachers & & & & & & & & & & & \\
\hline \multicolumn{13}{|c|}{ Perception of social support } \\
\hline $0-11$ & Not existent & & & & & & & & & & & \\
\hline $12-22$ & $\begin{array}{l}\text { Not a pleasant } \\
\text { place }\end{array}$ & & & & & & & & & & & \\
\hline $23-33$ & $\begin{array}{l}\text { More pros than } \\
\text { cons }\end{array}$ & 26.9 & 24.4 & 24.2 & 25.1 & 24.1 & 26.6 & 30.3 & 27.5 & 29.0 & 23.8 & $\begin{array}{l}25.8 \\
(5.6)\end{array}$ \\
\hline $34-44$ & $\begin{array}{l}\text { A good supportive } \\
\text { environment }\end{array}$ & & & & & & & & & & & \\
\hline \multicolumn{13}{|l|}{ Overall } \\
\hline $0-40$ & Very poor & & & & & & & & & & & \\
\hline $41-80$ & $\begin{array}{l}\text { Plenty of } \\
\text { problems }\end{array}$ & & & & & & & & & & & \\
\hline $80-120$ & $\begin{array}{l}\text { More positive } \\
\text { than negative }\end{array}$ & 100.3 & 88.8 & 99.6 & 104.1 & 90.1 & 99.2 & 115.1 & 105 & 102 & 90.6 & $\begin{array}{l}98.3 \\
(20.0)\end{array}$ \\
\hline $121-160$ & Excellent & & & & & & & & & & & \\
\hline
\end{tabular}

Table 4. Items rated below average by the different specialties

\begin{tabular}{ll}
\hline Specialty & Items with scores $<\mathbf{2 . 0}$ \\
\hline Dental surgery & - \\
Ear, nose and throat & 17,20 \\
General outpatient department & 20 \\
Medicine & 20,25 \\
Obstetrics and gynaecology & $17,19,20,32$ \\
Ophthalmology & - \\
Paediatrics & $20,23,25,36$ \\
Pathology & 20,25 \\
Radiology & 20 \\
Surgery & 17
\end{tabular}

have good collaboration with other junior doctors'). The majority of the items (31 of 40) had mean scores between 2 and 3, with only four items scoring $>3(7,13,16$ and 29). These results highlight that the majority of the areas in the learning environment need improvement. However, it is also good to know that the areas where the junior doctors were most satisfied with their environment border on racism and tribalism, sex discrimination, collaboration with other junior doctors and sense of belonging to a team. In a study by Vieira, ${ }^{[15]}$ it was noted that the residents also had a positive perception of the learning environment in terms of gender and racism. Clapham et al. ${ }^{[16]}$ reported similar findings in a small sample of intensivecare residents in a hospital in the UK.

Weaknesses identified in the environment, as indicated by a mean score of $<2$, appeared in five items $(9,20,26,32$ and 38). This signifies that there was no information booklet available for junior doctors to orientate them with regard to the postgraduate programme. Moreover, other weaknesses 
Table 5. Analysis of the differences in item scores among the specialties

\begin{tabular}{llll}
\hline Item number & Statement & $\boldsymbol{F}$-value & $\boldsymbol{p}$-value \\
\hline 5 & I have the appropriate level of responsibility in this post & 2.545 & 0.011 \\
9 & There is an informative junior doctors' handbook & 3.096 & 0.003 \\
14 & There are clear clinical protocols in this post & 3.551 & 0.001 \\
17 & My hours of work conform to the civil service rule & 3.023 & 0.003 \\
26 & There are adequate catering facilities when I am on call & 2.498 & 0.013 \\
32 & My workload in this job is fine & 5.559 & $<0.001$ \\
35 & My clinical teachers have good mentoring skills & 2.489 & 0.013 \\
39 & My clinical teachers provide me with good feedback on my strengths and weaknesses & 1.999 & 0.047
\end{tabular}

were: inadequate accommodation and catering facilities when on call, excessive workload and absence of good counselling opportunities. Vieira ${ }^{[15]}$ reported low scores in four of these areas, suggesting lack of professional and personal support in the learning environment. Similarly, Al-Sheikh et al. ${ }^{[14]}$ reported the lowest scores for catering, housing, information and guidance. The absence of adequate orientation of junior doctors at the beginning of their training, as seen with the low score for item 9, may have an effect on their learning, as this could result in difficulties in making informed choices in career paths from peculiarities of the different clinical rotations. An information handbook detailing the job description for every member of the health team could reduce the possibility of exploitation. ${ }^{[14]}$

It is interesting to note that three of these items (20, 26 and 38) are in the domain of the perception of social support. The results of these items revealed that the educational environment is weak in the area of social support. There was general agreement among the departments with regard to item 20 ('This hospital has good-quality accommodation for junior doctors, especially when on call') as 9 of the 10 specialties scored it $<2.0$ (Table 4). These three items in the social support domain, and the low score for item 32 ('My workload in this job is fine'), may support the association between the residency training programme and stress, depression and burnout, which are thought to be mainly due to excessive working hours, sleep deprivation, challenging patients and an aggressive and challenging work environment. ${ }^{[17-19]}$ To improve the learning environment of the doctors in this study, attention should be given to the following focus areas: developing and providing the junior doctors with an information handbook, improving the quality of the accommodation, improving the catering facilities, finding a way to reduce the workload, and providing counselling for the junior doctors if needed. Lleras and Durante, ${ }^{[20]}$ in a recent study, found a significant negative correlation between the educational environment and burnout among resident doctors, using PHEEM and Maslach Burnout Inventory questionnaires. The approach to reducing burnout in residents should include other components of the working environment, as a reduction in the workload alone has been found to be unsuccessful. $^{[21]}$

Analysis of the overall scores and subscale scores for level of training, gender and specialty department revealed no significant difference in the overall and subscale scores. Khoja ${ }^{[22]}$ reported a significantly better perception of the learning environment by the junior doctors in their last year of training compared with those in the first 3 years. Possible reasons were reduced workload, greater contact time with their trainers, and increased supervision and feedback from their trainers. However, Clapham et al. ${ }^{[16]}$ found that senior house officers scored the learning environment better than other junior doctors who are ahead of them in training. Boor et al., with similar results as Clapham et al., ${ }^{[16]}$ explained that the house officers and senior house officers may have a better perception of the learning environment, as they have lesser responsibilities and stress compared with those faced by other junior doctors who are ahead of them in training. The differences noted in the results of the abovementioned studies may be due to varying regulations regarding duties and workload for the levels of trainees in different institutions. Kanashiro et al., ${ }^{[23]}$ as in the current study, did not find significant differences in the perception of the learning environment among the house officers, senior house officers and the other junior doctors who are ahead of them in training level. However, the authors noted significant gender differences in the scores. Our study and other studies ${ }^{[12-14]}$ did not find significant gender differences in PHEEM scores.

Although all the specialties or departments were in the same hospital, there was a significant difference in their perceptions of some aspects of the learning environment, as seen, for example, with items 9,17 , and 32 , where the junior doctors in dental surgery, internal medicine, ophthalmology, radiology, paediatrics and obstetrics and gynaecology had significantly different perceptions, respectively, than their counterparts in other specialties. These differences may reflect variations in the organisation and use of set protocols in the departments, as well as staff strength and workload assigned to individuals. Some specialties may make increased demands on the time of the junior doctors. This may explain why junior doctors in paediatrics and obstetrics and gynaecology reported a significantly greater level of disagreement with their workload. Algaidi ${ }^{[24]}$ also noted differences in the perceptions of the learning environment among specialties in the same hospital, where he reported a significantly better perception of the learning environment by the junior doctors in general surgery compared with those in internal medicine. According to Algaidi, ${ }^{[24]}$ this is an indication that the learning environment in each department is as important as the general hospital environment, which may have significant effects on the quality of the learning environment.

\section{Conclusion}

This study has identified areas of strengths and weaknesses in the hospital educational environment and the 10 individual specialty departments in the same hospital. Interventions in the areas of weaknesses identified should be implemented and followed up with regular assessments of the educational environment using PHEEM as a means of quality control. Although this study presented findings from only one hospital, the results could assist other curriculum developers in the country's postgraduate medical training programmes in assessing their learning environment, making comparisons 
and introducing measures to improve the environment for the training of junior doctors. This is important, as the quality of the junior doctors' experiences in the learning environment relates to the quality of training and therefore the quality of care received by the patients they care for.

\section{Acknowledgements. None.}

Author contributions. PII: study idea, development of the study idea, experimental design, and preparation of the manuscript; KIS: collected data, evaluated statistics and contributed to discussion; HOO: further development of the study idea, development of experimental design and proofreading of the manuscript; ZM: proofreading of the manuscript and development of experimental design; and HMA: collected data, prepared results from statistical evaluation and contributed to manuscript preparation.

\section{Funding. None.}

\section{Conflicts of interest. None.}

1. West African Postgraduate Medical College. Profile of the college. http://www.wapmc.org/about (accesse 18 February 2016).

2. National Postgraduate Medical College of Nigeria. President's speeches: Welcome NPMCN. http://www.npmcn. edu.ng/welcome-to-npmen (accessed 18 February 2016).

3. Chambers R, Wall D. Teaching Made Easy: A Manual for Health Professionals. Abingdon, UK: Radcliffe Medical Press, 2000

4. Daugherty SR, Baldwin DC Jr, Rowley BD. Learning, satisfaction, and mistreatment during medical internship: A national survey of working conditions. JAMA 1998;279(15):1194-1199. https://doi.org/10.1001/jama.279.15.1194 5. Shimizu T, Tsugawa Y, Tanoue Y, et al. The hospital educational environment and performance of residents in the general medicine in-training examination: A multicenter study in Japan. Int J Gen Med 2013;6(1):637-640. https://doi.org/10.2147/IJGM.S45336

6. Boor K, Scheele F, van der Vleuten CP, Scherpbier AJ, Teunissen PW, Sijtsma K. Psychometric properties of an instrument to measure the clinical learning environment. Med Educ 2007;41(1):92-99. https://doi.org/10.1111 j.1365-2929.2006.02651.

7. Hoff TJ, Pohl H, Bartfield J. Creating a learning environment to produce competent residents: The roles of culture and context. Acad Med 2004;79(6):532-539. https://doi.org/10.1097/00001888-200406000-00007

8. Kendall ML, Hesketh EA, Macpherson SG. The learning environment for junior doctor training - what hinders, what helps. Med Teach 2005;27(7):619-624. https://doi.org/10.1080/01421590500069710
9. Cross V, Hicks C, Parle J, Field S. Perceptions of the learning environment in higher specialist training of doctors Implications for recruitment and retention. Med Educ 2006;40(2):121-128. https://doi.org/10.1111/j.13652929.2005.02382.x

10. Wall D, Clapham M, Riquelme A, et al. Is PHEEM a multi-dimensional instrument? An international perspective. Med Teach 2009;31(11):e521-e527. https://doi.org/10.3109/01421590903095528

11. Rotem A, Bloomfield L, Southon G. The clinical learning environment. Isr J Med Sci 1996;32(9):705-710.

12. Roff S, McAleer S, Skinner A. Development and validation of an instrument to measure the postgraduate clinical learning and teaching environment for hospital-based junior doctors in the UK. Med Teach 2005;27(4):326-331. https://doi.org/10.1080/01421590500150874

13. Aspegren K, Bastholt L, Bested KM, et al. Validation of the PHEEM instrument in a Danish hospital setting. Med Teach 2007;29(5):498-500. https://doi.org/10.1080/01421590701477357

14. Al-Sheikh MH, Ismail MH, Al-Khater SA. Validation of the postgraduate hospital educational environment measure at a Saudi university medical school. Saudi Med J 2014;35(7):734-738.

15. Vieira JE. The postgraduate hospital educational environment measure (PHEEM) questionnaire identifie quality of instruction as a key factor predicting academic achievement. Clinics 2008;63(6):741-746. https://do org/10.1590/S1807-59322008000600006

16. Clapham M, Wall D, Batchelor A. Educational environment in intensive care medicine: Use of Postgraduate Hospital Educational Environment Measure (PHEEM). Med Teach 2007;29(6):184-191. https://do org/10.1080/01421590701288580

17. Al-Marshad S, Alotaibi G. Evaluation of clinical education environment at King Fahad Hospital of Damman University using the Postgraduate Hospital Education Environment Measure (PHEEM) Inventory. Educ Med 2011;3(2):e6-e14. https://doi.org/10.5959/eimj.3.2.2011.or1

18. Collier VU, McCue JD, Markus A, Smith L. Stress in medical residency: Status quo after a decade of reform? Ann Intern Med 2002;136(5):384-390. https://doi.org/10.7326/0003-4819-136-5-200203050-00011

19. Peterlini M, Tiberio IF, Saadeh A. Pereira JC, Martins MA. Anxiety and depression in the first year of medical Peterlini M, Tiberio IF, Saadeh A, Pereira JC, Martins MA. Anxiety and depression in the first ye
residency training. Med Educ 2002;36(1):66-72. https://doi.org/10.1046/j.1365-2923.2002.01104.X

20. Lleras J, Durante E. Correlation between the educational environment and burn-out syndrome in residency programs at a university hospital. Arch Argent Pediatr 2014;112(1):e6-e11. https://doi.org/10.5546/aap.2014.eng.6 1. Celfand DV, Podnos YD, Carmichael JC, Saltzman DJ, Wilson SE, Williams RA. Effect of the 80-hour workweek on resident burnout. Arch Surg 2004;139(9):933-938. https://doi.org/10.1001/archsurg.139.9.933

22. Khoja AT. Evaluation of the educational environment of the Saudi family medicine residency training program. J Fam Community Med 2015;22(1):49-56. https://doi.org/10.4103/2230-8229.149591

23. Kanashiro J, McAleer S, Roff S. Assessing the educational environment in the operating room - a measure of resident perception at one Canadian institution. Surgery 2006;139(2):150-158. https://doi.org/10.1016/ surg.2005.07.005

24. Algaidi SA. Assessment of educational environment for interns using Postgraduate Hospital Educationa Environment Measure (PHEEM) J J U: Med Sci 2010;5(1):1-12. https://doi.org/10.1016/s1658-3612(10)70118-9 